\title{
An Improved Fast Photochemical Oxidation of Proteins (FPOP) Platform for Protein Therapeutics
}

\author{
Ying Zhang, Don L. Rempel, Hao Zhang, Michael L. Gross \\ Department of Chemistry, Washington University in St. Louis, St. Louis, MO 63130, USA
}

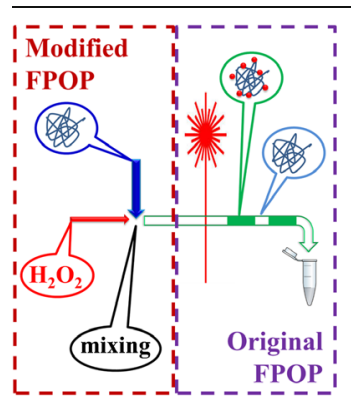

\begin{abstract}
Unlike small-molecule drugs, the size and dynamics of protein therapeutics challenge existing methods for assessing their high order structures (HOS). To extend fast photochemical oxidation of proteins (FPOP) to protein therapeutics, we modified its platform by introducing a mixing step prior to laser irradiation to minimize unwanted $\mathrm{H}_{2} \mathrm{O}_{2}$-induced oxidation. This improvement plus standardizing each step yield better reproducibility as determined by a fitting process whereby we used a nonFPOP spectrum as a template to report the unmodified level. We also tested different buffer systems for this modified FPOP platform with cytochrome $c$. The outcome is a standard oxidation profile that can be compared between different laboratories and regulatory agencies that wish to adopt FPOP for quality control purposes.
\end{abstract}

Key words: Fast photochemical oxidation of proteins, FPOP, Improved platform, Cytochrome $c$, Asymmetric mixer, Protein therapeutics

Received: 22 October 2014/Revised: 19 November 2014/Accepted: 20 November 2014/Published Online: 18 December 2014

\section{Introduction}

$\mathrm{T}$ he development of protein therapeutics, especially monoclonal antibodies (mAbs), continues to grow [1-3]. Unlike traditional small-molecule therapeutics (up to $500 \mathrm{Da}$ ), mAbs are macromolecules that contain over 1000 amino acids, require comprehensive biochemical and biophysical characterization not only of the primary but also, more challengingly, of the high order structures (HOS) [4-6]. HOS can be characterized by size exclusion chromatography (SEC), capillary electrophoresis (CE), X-ray crystallography (X-ray), and nuclear magnetic resonance (NMR) [7]. With the exception of X-ray and NMR, most approaches afford limited site-specific information. X-ray and NMR provide high resolution structures but at the cost of time and sample. Needed are new approaches with fast turnaround and low sample consumption [8].

Mass spectrometry (MS)-based top-down and bottom-up approaches now show effectiveness for characterization of $\mathrm{mAb} \operatorname{HOS}[4,9,10,6]$. Although top-down strategies for mAbs or other protein therapeutics $[11,12]$ are emerging, a more general strategy is to use hydrogen deuterium exchange

Electronic supplementary material The online version of this article (doi:10.1007/s13361-014-1055-0) contains supplementary material, which is available to authorized users.

Correspondence to: Michael Gross; e-mail: mgross@wustl.edu
(HDX) or FPOP and bottom-up sequencing [13-15] to pinpoint changes in HOS. Knowledge of these HOS changes must be known to guarantee drug quality.

FPOP utilizes highly reactive hydroxyl radicals, which are generated by laser photolysis of $\mathrm{H}_{2} \mathrm{O}_{2}$, to modify oxidatively the side chains of more than half the common amino acids [16]. Reactivity is determined by solvent accessibility and intrinsic reactivity, providing a measure for HOS. Unlike HDX, FPOP can use slow and effective separation and analysis without erasing modification information. The peptides carrying oxidation are more hydrophilic than the unmodified, making them easier to detect by LC/MS. Moreover, the FPOP platform can be built in most laboratories unlike the sychrotron method [17, 18]. Thus, FPOP is complementary to HDX for assuring correct HOS of protein therapeutics [19, 20].

A modified FPOP platform is needed to meet the stringent requirements of protein therapeutics characterization. One modification described here is to premix the $\mathrm{H}_{2} \mathrm{O}_{2}$ with the protein solution just seconds prior to laser irradiation to minimize $\mathrm{H}_{2} \mathrm{O}_{2}$-induced oxidation. In addition, the time interval between sample preparation and laser irradiation is readily controlled, and this should increase measurement precision. Furthermore, effects of different buffers in various FPOP experiments need to be normalized before comparison.

Herein, we describe a modified FPOP platform for protein therapeutics in which we admit a test protein, cytochrome $c$ (cyt. c), in buffer into the FPOP device. Cyt. $c$ is chosen 
because it has a redox-active iron-heme center and, thus, is sensitive to oxidation. Using cyt. $c$, we demonstrated the reproducibility and robustness of this modified FPOP platform under demanding conditions. We also added an asymmetrical mixing step just prior to laser irradiation to minimize protein exposure to $\mathrm{H}_{2} \mathrm{O}_{2}$; others have made similar developments, but the motivation was to study protein folding [21]. The outcome of our study is improved precision of footprinting. This modified platform should be useful particularly in pharmaceutical applications, where reproducibility and integrity of data for protein therapeutics are major issues.

\section{Experimental}

$\mathrm{H}_{2} \mathrm{O}_{2}$, water, acetonitrile, and formic acid at the highest purity available were obtained from Sigma-Aldrich (St. Louis, MO, USA). Phosphate-buffered saline (PBS) powder, Trizma base, catalase from bovine liver, bovine cyt. $c$ were also from SigmaAldrich. The cyt. $c$ stock solution was prepared by filtering with a micro bio-spin 6 column (Bio-Rad, Hercules, CA, USA).

A $248 \mathrm{~nm} \mathrm{KrF}$ excimer laser (GAM Laser Inc., Orlando, FL, USA) tuned to approximately $30 \mathrm{~mJ} /$ pulse was used to irradiate the sample solution. The laser beam was focused with a convex lens (Edmunds Optics, Barrington, NJ, USA) onto a $150 \mu \mathrm{m}$ i.d. fused silica (Polymicro Technologies, Pheonix, AZ, USA), giving a 2.5-3.0 mm irradiation window. The laserpulse frequency was controlled by an external pulse generator (B\&K Precision, Yorbal Linda, CA, USA). The platform was a modified version of our previous FPOP setup [16]. $\mathrm{H}_{2} \mathrm{O}_{2}$ and the scavenger (glutamine, Gln) in PBS or Tris buffer were mixed with protein $(15 \mu \mathrm{M})$ solutions just prior to the FPOP irradiation window. The mixing involved inserting the silica tubing into a micro-tee mixer (Cobert Associates Lab, St. Louis, MO, USA) and was achieved by using different i.d. (for example, 75 and $150 \mu \mathrm{m}$ ) of silica tubing (Polymicro Technologies, Pheonix, AZ, USA) with different flow rates. The flow rates were also adjusted to ensure a $15 \%$ exclusion volume to avoid repeated $\cdot \mathrm{OH}$ reaction. The labeled sample was collected in low-binding tubes containing $10 \mu \mathrm{L}$ of $50 \mathrm{nM}$ catalase and $200 \mathrm{mM}$ methionine to decompose residual $\mathrm{H}_{2} \mathrm{O}_{2}$.

The labeled protein samples were analyzed as described previously [22]. They were desalted with a C8 trap column using a short gradient. All ESI mass spectra were acquired in the positive-ion mode on a MaXis $4 \mathrm{G}$ quadrupole time-offlight (Q-Tof) mass spectrometer (Bruker, Bremen, Germany).

\section{Results and Discussion}

Goals of developing a modified FPOP platform are to minimize the interference of $\mathrm{H}_{2} \mathrm{O}_{2}$-induced oxidation of the protein, maximize that due to $\bullet \mathrm{OH}$, and improve the reproducibility to help meet requirements for pharmaceutical applications. Previously in FPOP, the protein solution is pre-incubated with $\mathrm{H}_{2} \mathrm{O}_{2}$ and Gln and loaded into a syringe that flows the solution in a tube past an FPOP window. The incubation time of the proteins in such solutions can be 2-3 min or longer depending on experimental requirements and unplanned difficulties. $\mathrm{H}_{2} \mathrm{O}_{2}$ induced oxidative modification can occur on highly reactive side chains of methionine and cysteine during this time, possibly introducing conformational changes to the protein and adding ambiquity to results.

To address this issue, we incorporated an asymmetrical mixing step prior to laser-irradiation window (Figure 1). With the improvement, the protein solution in its original buffer is delivered using one syringe, whereas $\mathrm{H}_{2} \mathrm{O}_{2}$ and Gln are delivered with another to give mixing at a set ratio (2:1 protein: $\mathrm{H}_{2} \mathrm{O}_{2} / \mathrm{Gln}$ solution) seconds prior to laser irradiation. The asymmetric mixing assures that it is complete in less than $1 \mathrm{~s}$.

The second improvement for the FPOP platform is to increase the measurement precision for the extents of oxidation from experiment to experiment. We standardized each step in the modified platform, from initiating the syringe pump to collecting the samples following FPOP by controlling time accurately. Another improvement is that collecting the "plugs" of oxidized protein solution is delayed by $60 \mathrm{~s}$ to ensure the stability of syringe pumping and laser irradiation. Any solution and air bubbles that remain in the capillary following washing are also pushed out so that the concentrations of the protein, $\mathrm{H}_{2} \mathrm{O}_{2}$, and $\mathrm{Gln}$ do not change, thus eliminating other variability. For example, the laser irradiation starts at time 0 ; the syringe pump pushing $\mathrm{H}_{2} \mathrm{O}_{2}$ and $\mathrm{Gln}$, protein solutions, and the collection starts $20-80 \mathrm{~s}$ later, respectively. The time for collecting a sample is 120 to $150 \mathrm{~s}$, depending on the laser window width, laser frequency and the exclusion volume. This time is constant, however, for each experiment. In summary, all the FPOP experiments with this platform were controlled by a timer on the sec timescale. This standardized process guarantees each sample to be treated nearly identically, reducing intersample variations.

We used a model protein, cyt. $c$, to evaluate the modified FPOP platform. We chose to conduct three experiments: nonlaser control, non- $\mathrm{H}_{2} \mathrm{O}_{2}$ control, and FPOP, by following the modified protocol. Both non-laser and non- $\mathrm{H}_{2} \mathrm{O}_{2}$ controls in which all the steps were the same except the laser was not fired

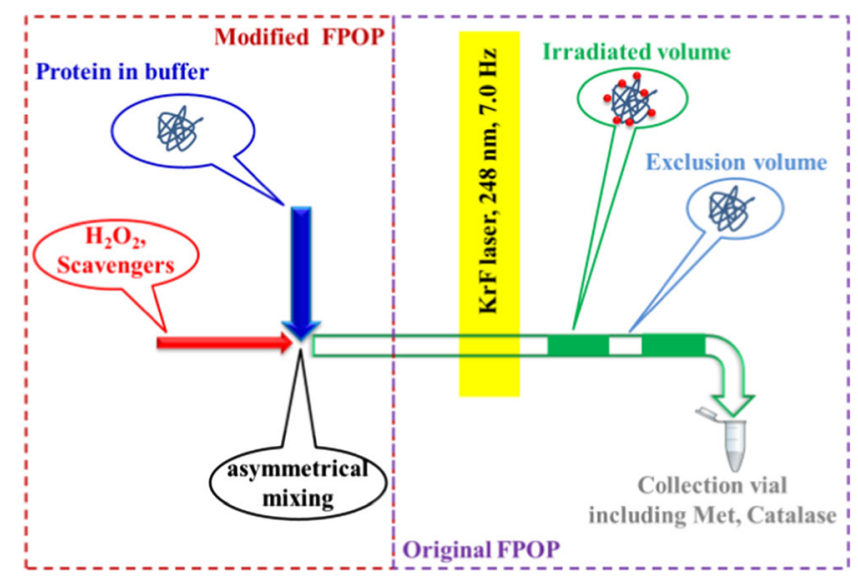

Figure 1. Scheme for the modified FPOP platform 
or the $\mathrm{H}_{2} \mathrm{O}_{2}$ was not added, respectively, afforded the oxidative baseline for the modified FPOP platform. Each experiment was performed in quadruplicate to afford a good evaluation of precision (Figure 2). We recommend that an oxidation profile of the model protein under various FPOP conditions be used to benchmark any platform and assure both reproducibility and an appropriate level of oxidation for new experiments.

We then analyzed the intact, non-digested samples following fast desalting to obtain a quick picture of the nature and extent of oxidation. Although multiply charged protein ions were observed (from +8 to +21 ) within 0.3 min elution window from the trapping column, we chose the most abundant charge state $(+15)$ to give the oxidation profile (Figure 2$)$ at good S/N, and we integrated the signals over the entire elution window. Each species was identified with a mass error less than $5 \mathrm{ppm}$. For each charge state, the most intense peak corresponds to the unmodified protein species. There is a lower intensity peak corresponding to the singly oxidized species $(+16 \mathrm{Da})$ observed for non-laser control experiments. Any multiply oxidized modified proteins (with one or more +16 Da shifts) were only observed in the full FPOP experiments. We did not observe significant changes among various charge states, indicating robustness and good reproducibility (Figure S1, S2, and S3 for $+9,+12$ charges, and deconvoluted spectra, respectively).

We then fit the mass spectra by using a custom MathCADbased (ver. 14.0 M010 by Parametric Technology Corporation, Needham, MA, USA) fitting program as described in Supporting Information. The \% unmod results for all nonlaser controls and non- $\mathrm{H}_{2} \mathrm{O}_{2}$ controls (Figure 2) show that only small amounts of modification are seen. With FPOP, multiple

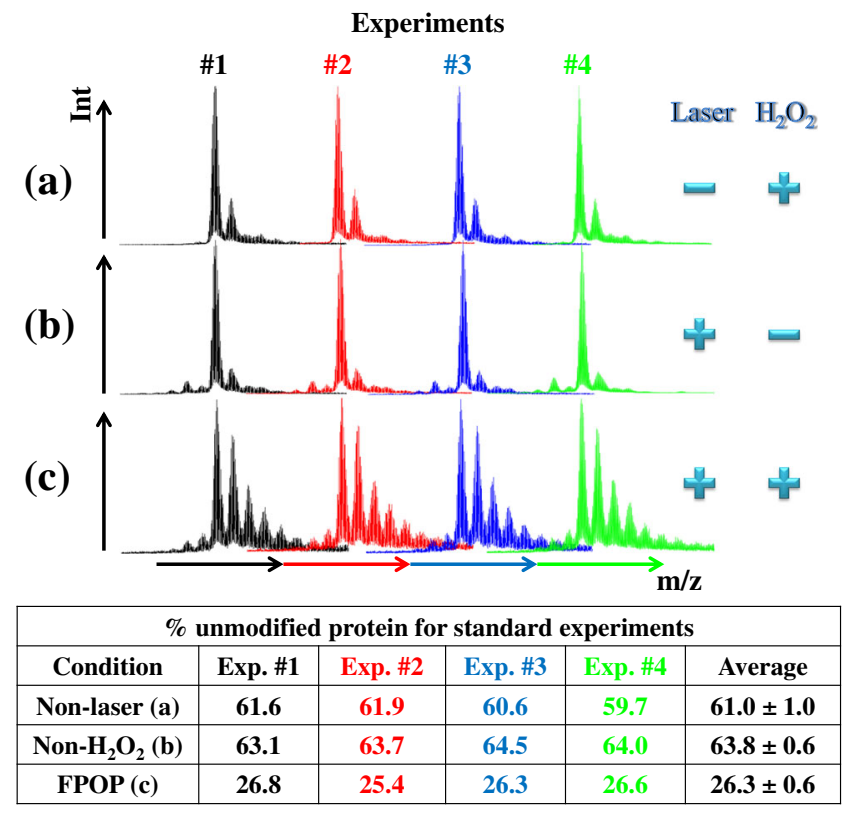

Figure 2. Mass spectra of FPOP-labeled cyt. $c$ (+15 charge) obtained by using a modified FPOP platform in quadruplicate for (a) "non-laser" control, (b) "non- $\mathrm{H}_{2} \mathrm{O}_{2}$ " control, and (c) standard FPOP conditions. The \% unmod for each experiment is included in the table accompanying the figure oxidation patterns were observed, and only $30 \%$ of the proteins under test conditions remain unmodified.

We compared the results obtained by submitting cyt. $c$ to the original FPOP setup (Figure 3) with those by the modified platform (in quadruplicate) to evaluate precision. For the original FPOP setup, the $\%$ unmod is $23 \% \pm 3 \%$, whereas the average is $26.3 \% \pm 0.6 \%$ with the modified ver. (Figure $3 \mathrm{~b}$ versus Figure $2 \mathrm{c})$. The improved precision $(0.6 \%$ versus $3 \%)$ demonstrates an advantage of the modified platform. In addition, the oxidation levels of products (i.e., +16 versus +32 species) also show greater variations when using the original FPOP setup. Furthermore, the unmodified level for non-laser condition is significantly lower with the original than with the modified platform ( $40 \%$ versus $61.0 \%$ ), suggesting $\mathrm{H}_{2} \mathrm{O}_{2}$-induced oxidation occurred with the original platform. This effect is diminished when FPOP occurs because it dominates the oxidation extent. Indeed, this oxidation may vary between proteins, depending on the protein, the number of solvent-accessible sites, and the number of readily oxidized residues (e.g., Met). For example, cyt. $c$ is only a $12 \mathrm{kDa}$ protein with most residues in flexible regions. Thus, its oxidation may be greater compared with a larger protein of a tighter structure (e.g., protein therapeutics). Incorporating a mixing step with $\mathrm{H}_{2} \mathrm{O}_{2}$ prior to FPOP establishes a more reproducible protocol with less non-FPOP oxidation.

Another advantage of FPOP is that the protein can be footprinted under conditions that are close either to physiological (unlike X-ray crystallography) or to those for storing/ administering protein therapeutics. Different buffer systems can introduce variations in the FPOP results because the buffer components can quench some of the radicals [23]. We tested PBS and Tris buffers to evaluate the effect. Different concentrations of Tris as well as of $\mathrm{H}_{2} \mathrm{O}_{2}$ were tested to achieve a similar oxidation profile as $10 \mathrm{mM}$ PBS and $15 \mathrm{mM} \mathrm{H}_{2} \mathrm{O}_{2}$ (Figure S4). With a higher concentration $(25 \mathrm{mM})$ of Tris buffer, the test protein undergoes $20 \%$ less oxidation (\% unmod

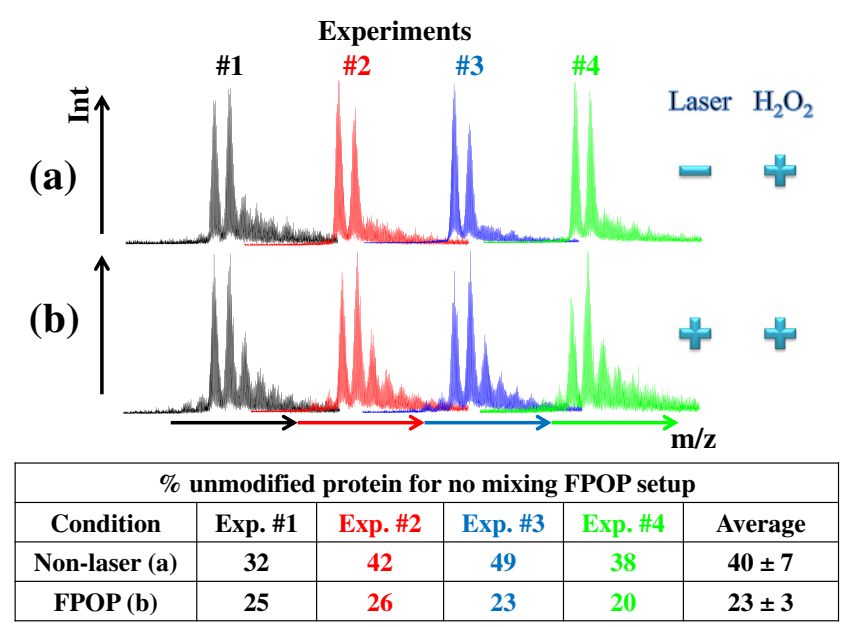

Figure 3. Mass spectra of FPOP-labeled cyt. $c$ using the original FPOP platform in quadruplicate for (a) "non-laser" control, and (b) standard FPOP conditions. The \% unmod for each experiment is included in the table accompanying the figure 
of $46.3 \%$ versus $26.3 \%$ ), or $10 \%$ when we used higher concentration $(30 \mathrm{mM})$ of $\mathrm{H}_{2} \mathrm{O}_{2}$ (\% unmod of $37.4 \%$ versus $26.3 \%$ ). Only when the protein was labeled in $10 \mathrm{mM}$ Tris buffer in the presence of $30 \mathrm{mM} \mathrm{H} \mathrm{H}_{2}$ can we obtain a similar oxidation profile as under typical conditions (\% unmod of $27.8 \%$ versus $26.3 \%$ ). These results suggest that the modified FPOP setup is flexible but needs normalization when using different buffers or setups (e.g., as in different laboratories).

In summary, the modified setup and experimental protocol give better reproducibility and less $\mathrm{H}_{2} \mathrm{O}_{2}$-induced oxidation, two improvements needed for protein therapeutics characterization and for FPOP in general. Different buffers ( $10 \mathrm{mM}$ Tris and $30 \mathrm{mM} \mathrm{H}_{2} \mathrm{O}_{2}$ ) provide similar profiles as the usual condition (10 mM PBS and $15 \mathrm{mM} \mathrm{H}_{2} \mathrm{O}_{2}$ ). We plan to test the improved platform by analyzing antibody-antigen systems.

\section{Acknowledgment}

This research was supported by the National Institute of General Medical Sciences (8 P41 GM103422) of the National Institutes of Health to M.L.G. The authors thank Aaron Wecksler, Patricia Molina, Galahad Deperalta, and Victor Ling of Genentech for support and helpful discussions.

\section{References}

1. Buss, N.A., Henderson, S.J., McFarlane, M., Shenton, J.M., de Haan, L.: Monoclonal antibody therapeutics: history and future. Curr. Opin. Pharmacol. 12(5), 615-622 (2012)

2. Nelson, A.L., Dhimolea, E., Reichert, J.M.: Development trends for human monoclonal antibody therapeutics. Nat. Rev. Drug Discov. 9(10), 767-774 (2010)

3. Stern, M., Herrmann, R.: Overview of monoclonal antibodies in cancer therapy: present and promise. Crit. Rev. Oncol./Hematol. 54(1), 11-29 (2005)

4. Beck, A., Wagner-Rousset, E., Ayoub, D., Van Dorsselaer, A., SanglierCianferani, S.: Characterization of therapeutic antibodies and related products. Anal. Chem. 85(2), 715-736 (2013)

5. Konermann, L., Vahidi, S., Sowole, M.A.: Mass spectrometry methods for studying structure and dynamics of biological macromolecules. Anal. Chem. 86(1), 213-232 (2014)

6. Zhang, Z., Pan, H., Chen, X.: Mass spectrometry for structural characterization of therapeutic antibodies. Mass Spectrom. Rev. 28(1), 147-176 (2009)

7. Lundblad, R.L.: Introduction to biopharmaceutical conformational analysis. In: Approaches to the conformational analysis of biopharmaceuticals. Protein Science. pp. 1-18. Chapman and Hall/CRC, Boca Raton, FL (2009)
8. Kaltashov, I.A., Bobst, C.E., Abzalimov, R.R., Wang, G., Baykal, B., Wang, S.: Advances and challenges in analytical characterization of biotechnology products: mass spectrometry-based approaches to study properties and behavior of protein therapeutics. Biotechnol. Adv. 30(1), 210222 (2012)

9. Bobst, C.E., Abzalimov, R.R., Houde, D., Kloczewiak, M., Mhatre, R., Berkowitz, S.A., Kaltashov, I.A.: Detection and characterization of altered conformations of protein pharmaceuticals using complementary mass spectrometry-based approaches. Anal. Chem. 80(19), 7473-7481 (2008)

10. Kaltashov, I.A., Bobst, C.E., Abzalimov, R.R.: Mass spectrometry-based methods to study protein architecture and dynamics. Protein Sci. 22(5), 530-544 (2013)

11. Tsybin, Y.O., Fornelli, L., Stoermer, C., Luebeck, M., Parra, J., Nallet, S., Wurm, F.M., Hartmer, R.: Structural analysis of intact monoclonal antibodies by electron transfer dissociation mass spectrometry. Anal. Chem. 83(23), 8919-8927 (2011)

12. Zhang, H., Cui, W., Wen, J., Blankenship, R.E., Gross, M.L.: Native electrospray and electron-capture dissociation FTICR mass spectrometry for top-down studies of protein assemblies. Anal. Chem. 83(14), 55985606 (2011)

13. Jones, L.M., Sperry, J.B., Carroll, J.A., Gross, M.L.: Fast photochemical oxidation of proteins for epitope mapping. Anal. Chem. 83(20), 7657-7661 (2011)

14. Wei, H., Mo, J., Tao, L., Russell, R.J., Tymiak, A.A., Chen, G., Iacob, R.E., Engen, J.R.: Hydrogen/deuterium exchange mass spectrometry for probing higher order structure of protein therapeutics: methodology and applications. Drug Discov Today. 19(1), 95-102 (2013)

15. Yan, Y., Chen, G., Wei, H., Huang, R.Y., Mo, J., Rempel, D.L., Tymiak, A.A., Gross, M.L.: Fast photochemical oxidation of proteins (FPOP) maps the epitope of EGFR binding to Adnectin. J. Am. Soc. Mass Spectrom. 25(12), 2084-2092 (2014)

16. Hambly, D.E., Gross, M.L.: Laser flash photolysis of hydrogen peroxide to oxidize protein solvent-accessible residues on the microsecond timescale. J. Am. Soc. Mass Spectrom. 16, 2057-2063 (2005)

17. Gupta, S., D'Mello, R., Chance, M.R.: Structure and dynamics of protein waters revealed by radiolysis and mass spectrometry. Proc. Natl. Acad. Sci. U. S. A. 109(37), 14882-14887 (2012)

18. Wang, L., Chance, M.R.: Structural mass spectrometry of proteins using hydroxyl radical based protein footprinting. Anal. Chem. 83(19), 72347241 (2011)

19. Jones, L.M., Zhang, H., Cui, W.D., Kumar, S., Sperry, J.B., Carroll, J.A., Gross, M.L.: Complementary MS methods assist conformational characterization of antibodies with altered S-S bonding networks. J. Am. Soc. Mass Spectrom. 24(6), 835-845 (2013)

20. Watson, C., Sharp, J.S.: Conformational analysis of therapeutic proteins by hydroxyl radical protein footprinting. AAPS J. 14(2), 206-217 (2012)

21. Vahidi, S., Stocks, B.B., Liaghati-Mobarhan, Y., Konermann, L.: Submillisecond protein folding events monitored by rapid mixing and mass spectrometry-based oxidative labeling. Anal. Chem. 85(18), 8618-8625 (2013)

22. Zhang, Y., Rempel, D.L., Zhang, J., Sharma, A.K., Mirica, L.M., Gross, M.L.: Pulsed hydrogen-deuterium exchange mass spectrometry probes conformational changes in amyloid beta (Abeta) peptide aggregation. Proc. Natl. Acad. Sci. U. S. A. 110(36), 14604-14609 (2013)

23. Xu, G., Chance, M.R.: Hydroxyl radical-mediated modification of proteins as probes for structural proteomics. Chem. Rev. 107(8), 3514-3543 (2007) 